\title{
TRACKING ROTATION AND TRANSLATION OF LASER MICROSURGICAL INSTRUMENTS
}

\author{
R. Ortiz and C. N. Riviere \\ The Robotics Institute, Carnegie Mellon University, Pittsburgh, PA 15213, USA
}

\begin{abstract}
An accurate optical sensing system has been developed to measure the position and orientation of a laser beam in two dimensions. The system is useful for evaluation of the accuracy of hand-held laser microsurgical instruments. The apparatus uses a lens and a beam-splitter to receive the incoming laser beam. Two position sensitive detectors placed at different distances from the beam splitter make it possible to rapidly and accurately calculate the position and orientation of the axis of the laser.
\end{abstract}

Keywords-Microsurgery, accuracy, optical sensing, position sensitive detector, angular measurement, tremor

\section{INTRODUCTION}

The need to improve accuracy in microsurgery has led to efforts to enhance accuracy using teleoperation $[1,2]$, the "steady-hand" approach in which surgeon and robot hold the same tool [3], and, most recently, a fully hand-held instrument developed in our laboratory to perform active tremor compensation [4]. Plans for this active instrument (known as "Micron")[5] include a prototype for hand-held laser microsurgery. To test this design, there is a need for a system to track the laser beam during tremor-canceling experiments.

There are many commercial systems that are commonly used in tracking surgical instruments, including Optotrak (Northern Digital, Waterloo, Canada), miniBird (Ascension Technology Corp., Burlington, Vt.), and Isotrack II (Polhemus, Colchester, Vt.). These systems offer sixdegree-of-freedom (6-dof) tracking and fast response, but, although their accuracy is high, it is still insufficient for microsurgical tremor studies. Given the small size of the active microsurgical instrument, another drawback is that they require that sensors be attached to the instrument, possibly resulting in a significant change in the very dynamics the experiments are designed to measure.

We have developed an instrument that uses a reflective approach to track the tip of Micron when fitted with a mechanical tool tip [6]. However, testing with the laserequipped instrument presents a slightly different problem. A single planar position sensitive detector (PSD), such as those used in [6], would allow tracking of the beam, but such a 2-dof sensing approach would not allow rotation to be distinguished from translation in the results. Actual localization of the laser axis in space is a 5-dof problem. However, one parameter can be determined and known by the design of the tracking instrument itself, leaving four parameters that must be tracked.
This paper presents the development of MADRID (Measurement Apparatus to Distinguish Rotational and Irrotational Displacement), a tracking instrument for laser microsurgical devices. It also presents initial results from calibration and testing of the instrument.

\section{METHODOLOGY}

\section{A. System development}

The primary elements in MADRID are optical sensors to track the laser beam. While CCD cameras could be used, this option is costly due to the two high-frame-rate and highresolution digital cameras and frame grabbers needed. As an alternative, PSDs offer high accuracy, high frequency response, and lower cost. They provide analog output, thus avoiding issues of pixel resolution, and are best suited to tracking a single light spot, such as in this application.

MADRID uses two PSDs to track the laser beam. A bandpass optical filter (10LF20-670, Newport, Irvine, CA) is used to block ambient light. The wavelength of the laser diode matches with the filter CWL (center wavelength). The bandwidth (FWHM) is $19.4 \mathrm{~nm}$. The laser is split in two beams by a Tech Spec ${ }^{\mathrm{TM}}$ Standard Cube Beam splitter (NT45-111, Edmund Optics, Barrington, NJ). The size of the cube is $12.5 \mathrm{~mm}$ on each side. After the split, each beam is received by a PSD (DL 100- 7PCBA, Pacific Silicon Sensor Inc., Westlake Village, CA). The DL 100-7PCBA is a duolateral position sensing module composed of a $1-\mathrm{cm}-$ square PSD and an associated amplifier circuit. It senses the position of a laser spot on the surface of the photodiode and gives analog current outputs indicating the centroid of the spot in $x$ and $y$, as well as intensity. The circuit converts that current signal into a voltage signal. In order to increase the linearity of the sensor, the PSD works in reverse bias, so the PSDs are powered by $\pm 15 \mathrm{~V}$ power supply. Under these conditions the linearity given by the PSD is \pm 1 percent of full scale.

Each sensor gives four outputs, two of which are related to the distance from the centroid of the light spot to the center of the PSD along the $x$-axis, while the other two deal with the $y$-axis. Within each output pair, one is proportional to the distance and the light intensity, while the other is proportional only to the intensity. By normalizing the $x$ and $y$ signal they become independent of the total light intensity and therefore independent of changes in the laser diode power. 
The analog signal is sampled at $1000 \mathrm{~Hz}$ with an A/D card (5508, ADAC, Woburn, Mass.). Every signal has an offset that is estimated and subtracted by software. Then the signals are passed through a software lowpass filter to reduce the noise.

The system is shown in Figure 1. The displacements measured by the two PSDs are the same when the laser beam is translated. If the beam is rotated (except for rotation about its own axis), the readings of the two PSDs will differ as a function of the difference between dimensions $a$ and $d$.

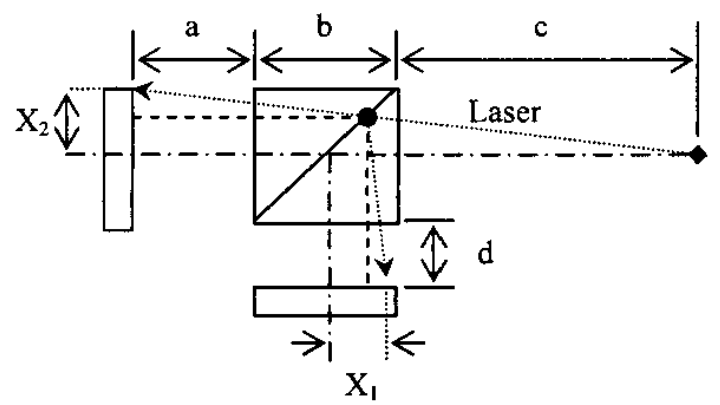

Fig. 1. Diagram of the device, showing how it can discriminate between translation and rotation. If $a \neq d$, then translations will affect each sensor equally, whereas rotations will produce unequal excursions on the two sensors. follows.

Assuming small angles, the rotations are computed as

$$
\begin{aligned}
& \alpha=\operatorname{Arctan}\left(\frac{x_{1}-x_{2}}{a-d}\right) \\
& \beta=\operatorname{Arctan}\left(\frac{y_{1}-y_{2}}{a-d}\right)
\end{aligned}
$$

In practice, since the quantity $(a-d)$ is known, the values of $x_{l}, y_{l}, \alpha$, and $\beta$ are sufficient to fully determine the line that represents the laser beam axis, though the actual distance, $c$, to the laser diode is unknown.

If $c$ is known, the position, $g$, of the laser diode can be calculated as follows.

$$
\begin{aligned}
& g_{x}=x_{1}-(d+b+c) \tan \alpha \\
& g_{y}=y_{1}-(d+b+c) \tan \beta
\end{aligned}
$$

The values of $a, b$, and $d$ are known to some accuracy, being part of the design, and can be estimated with greater precision during the calibration process. The workspace of the system depends on the size of the PSD $\left(1 \mathrm{~cm}^{2}\right.$, in this system) and on $c$.

\section{A. Experimental methods}

MADRID has been calibrated, tested for quantification of noise, and then used to track a laser held in the human hand. Precision micrometer stages were used for calibration. Each point used in the calibration was obtained by averaging 4000 points sampled at a rate of $1000 \mathrm{~Hz}$.

Translational calibration was performed with $\alpha$ and $\beta$ held equal to zero. The laser was moved in steps of $1 \mathrm{~mm}$. This first calibration allowed determination of the misalignment in the assembly.

After calibration, a performance test was done with the laser moving in both $x$ and $y$ in steps of $50 \mu \mathrm{m}$ for 20 steps, for total travel of $1 \mathrm{~mm}$.

Rotational calibration was then performed. The laser was rotated using rotational stages, without translation. In the first pass, $\alpha$ was changed in steps of 3.82 arc minutes while $\beta$ was held constant. The angle $\beta$ was moved in steps of 6 arc minutes while $\alpha$ was held constant.

Following calibration, the mount for the laser was held motionless and a recording was taken. Using these data, samples of noise in $\alpha, \beta, g_{x}$, and $g_{y}$ were computed. A separate recording was then taken while a human subject attempted to hold the laser motionless in the hand.

\section{Results}

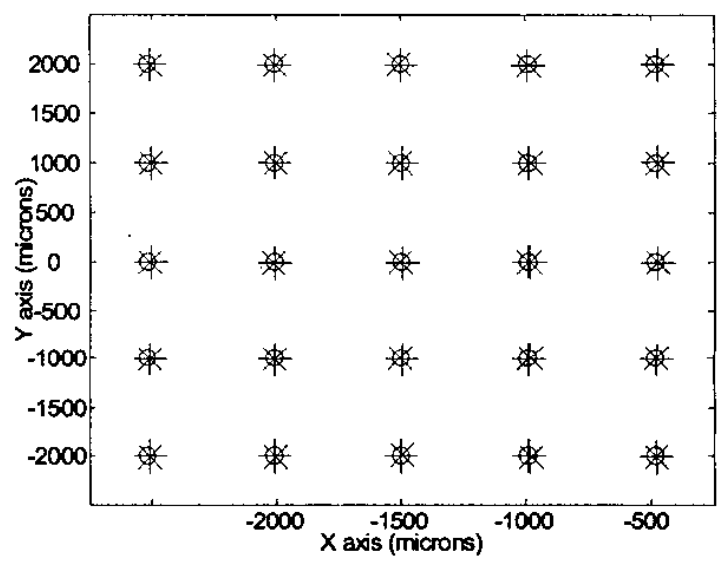

Fig. 2. Results from translational calibration. Circles represent target positions. Measurements from the first PSD are represented by + , and measurements from the second PSD by $X$.

Fig. 2 shows the result of transiational calibration. Circles represent the target positions, + represents the readings from the first $\mathrm{PSD}$, and $\times$ represents the readings from the second PSD. The maximum error in $x$ was 23.6 $\mu \mathrm{m}$, and the range measures $4064 \mu \mathrm{m}$. The nonlinearity is $0.58 \%$. The maximum error in $y$ is $20.1 \mu \mathrm{m}$, and the range measures $4000 \mu \mathrm{m}$. The nonlinearity is $0.50 \%$. 


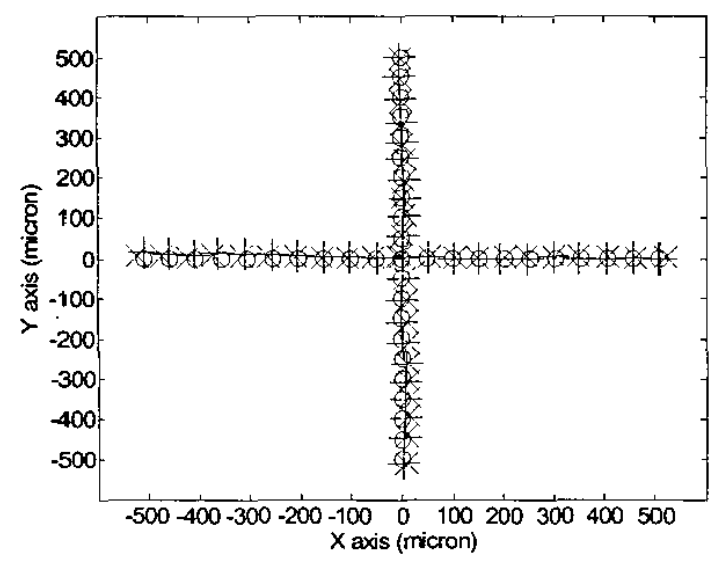

Fig. 3. Performance test: laser is moved in steps of $50 \mu \mathrm{m}$.

Figure 3 shows the results of the performance test. The maximum error in $x$ is $13.8 \mu \mathrm{m}$, and the range measures $1016 \mu \mathrm{m}$. The nonlinearity is $1.36 \%$. The maximum error in $y$ is $7.8 \mu \mathrm{m}$, and the range measures $1000 \mu \mathrm{m}$. The nonlinearity is $0.78 \%$.

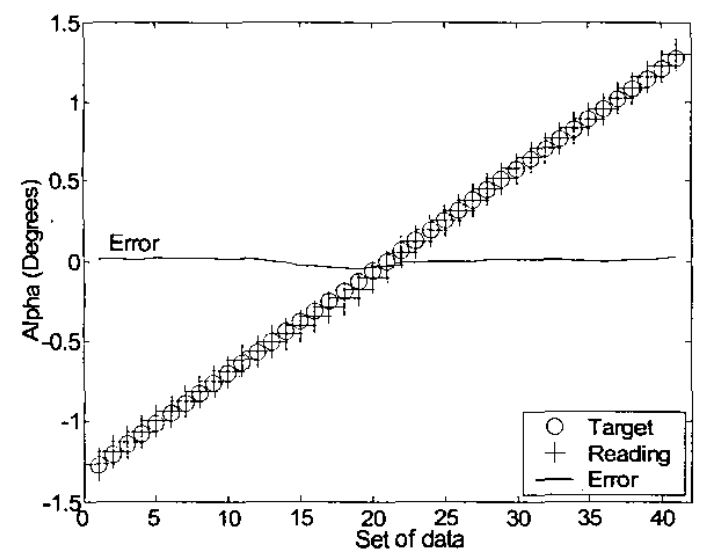

Fig. 4. Calibration test in one rotational degree of freedom $(\alpha)$.

Figure 4 presents the results of rotational calibration for

$\alpha$. The range of total rotation is $2.56^{\circ}$, maximum error is $0.048^{\circ}$, and error due to nonlinearity is $1.86 \%$.

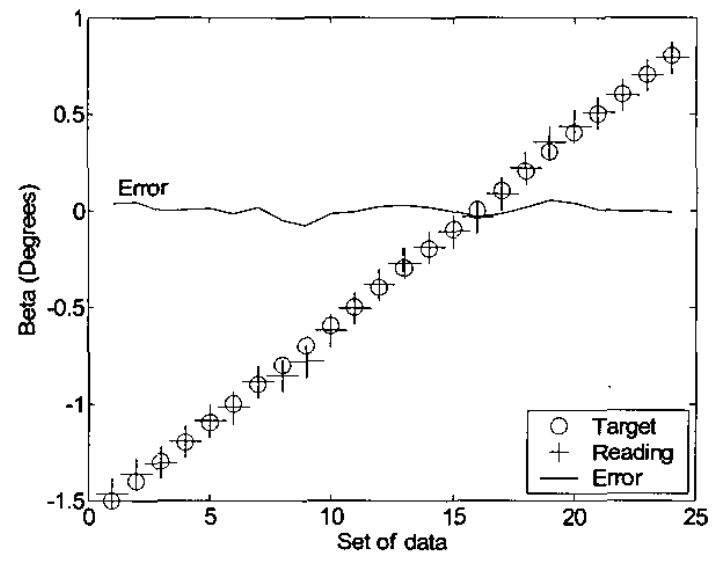

Fig. 5. Calibration test in one rotational degree of freedom $(\not)$.

Fig. 5 presents the results of calibration for $\beta$. The range of total rotation is $2.25^{\circ}$, maximum error is $0.077^{\circ}$, and error due to nonlinearity is $3.44 \%$.

Figs. 6 and 7 present the noise samples recorded with the laser mounted motionless on the bench top. The standard deviation of the noise is represented in Table I.
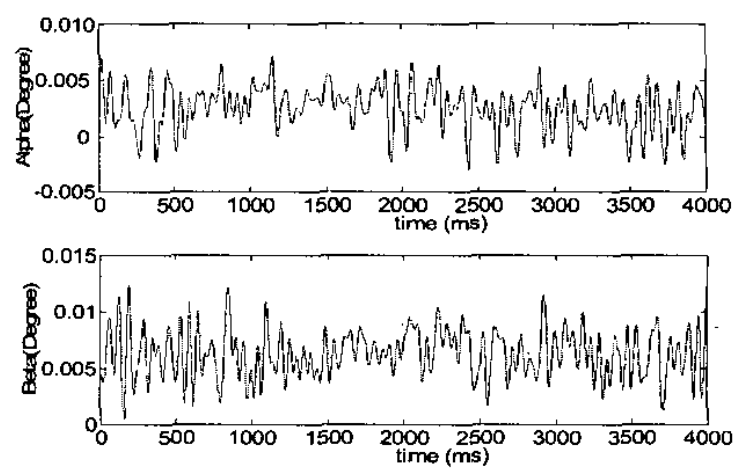

Fig. 6. Sample data recorded from a motionless laser, representing the noise of the system in rotation in $\alpha$ (top) and $\beta$ (bottom).
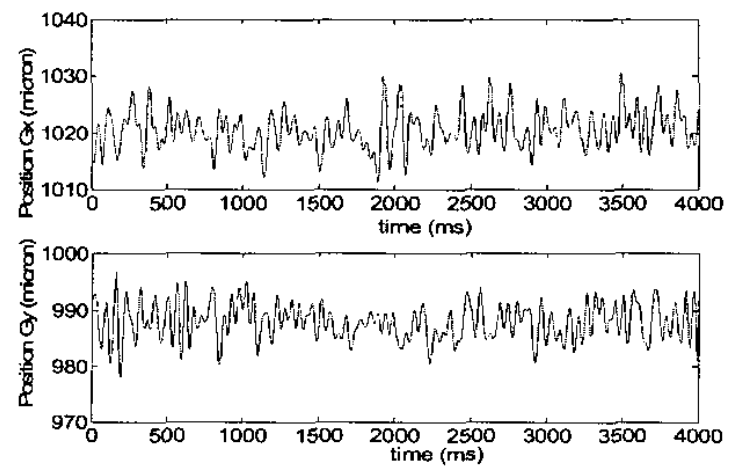

Fig. 7. Sample data recorded from a motionless laser, representing the noise of the system in translation in $x$ (top) and $y$ (bottom). 
TABLE I

Noise of the system.

\begin{tabular}{cc}
\hline DOF & Standard deviation \\
\hline$\alpha$ & $0.0022^{\circ} \approx 8^{\prime \prime}$ \\
$\beta$ & $0.0021^{\circ} \approx 8^{\prime \prime}$ \\
$g_{x}$ & $3.66 \mu \mathrm{m}$ \\
$g_{y}$ & $3.25 \mu \mathrm{m}$ \\
\hline
\end{tabular}

Figure 8 and 9 present the data recorded while a subject tried to hold the laser motionless in the hand.
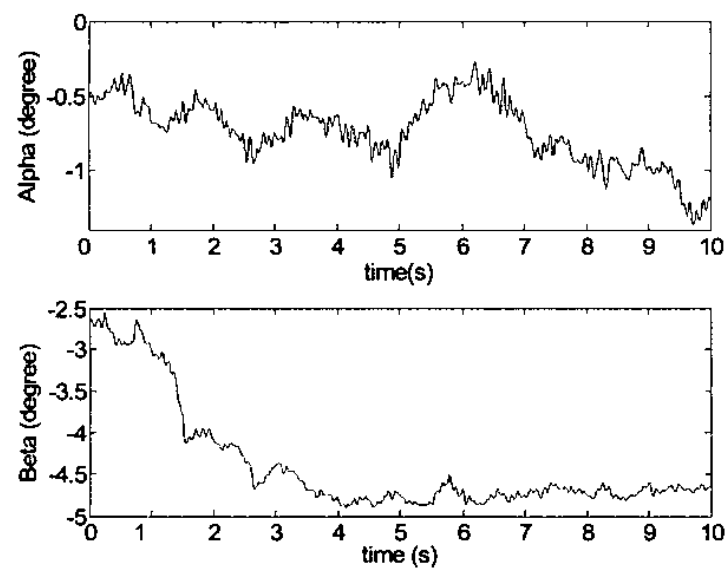

Fig. 8. Rotational data recorded while a subject attempted to hold a laser motionless in the hand, showing rotation in $\alpha$ (top) and $\beta$ (bottom).
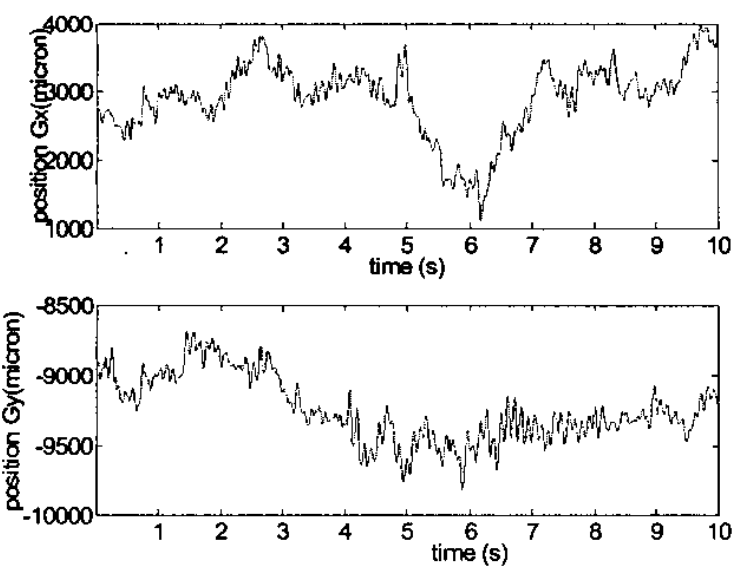

Fig. 9. Translational data recorded while a subject attempted to hold a laser motionless in the hand, shown movement in $x$ (top) and $y$ (bottom).

\section{DISCUSSION}

The results show that the system performs well enough to be used for evaluation of microsurgical instruments that are designed to achieve positioning accuracy of $10 \mu \mathrm{m}$. In addition to performance validation of mechatronic or robotic laser microsurgical instruments, the system is also useful for evaluation of the ergonomics of passive instruments, as well as for assessment of surgeons. Furthermore, it allows acquisition of high-precision data to be used in further development of error estimation algorithms such as those used in Micron.

\section{CONCLUSION}

An optical system to rack general motion (rotation and translation) of a laser beam has been developed using position sensitive detectors. The system will be used to validate the performance of hand-held mechatronic tools for laser microsurgery.

\section{ACKNOWLEDGMENT}

The authors would like to express their gratitude to $\mathrm{Mr}$. Wei Tech Ang for his constructive suggestions and technical assistance.

\section{REFERENCES}

[1] I. W. Hunter, T. D. Tilemachos, S. R. Lafontaine, P. G. Charette, L. A. Jones, M. A. Sagar, G. D. Mallinson, and P. J. Hunter, "A teleoperated microsurgical robot and associated virtual environment for eye surgery," Presence, vol. 2, pp. 265-280, 1993.

[2] H. Das, H. Zak, J. Johnson, J. Crouch, and D. Frambach, "Evaluation of a telerobotic system to assist surgeons in microsurgery," Computer Aided Surg., vol. 4, pp. 15-25, 1999.

[3] R. Taylor, P. Jensen, L. Whitcomb, A. Barnes, R. Kumar, D. Stoianovici, P. Gupta, Z. Wang, E. de Juan, Jr., and L. Kavoussi, "A steady-hand robotic system for microsurgical augmentation", Lecture Notes in Computer Science, Springer-Verlag, vol. 1679, pp.1031-1041, 1999

[4] C. N. Riviere, W. T. Ang, and P. K. Khosla, "Toward active tremor canceling in hand-held microsurgical instruments," IEEE Trans. Robot. Autom., 2003, in press.

[5] W. Ang, P. Khosla, and C. Riviere, "Design and implementation of active error canceling in a hand-held microsurgical instrument," Proc. IEEE/RSJ Int. Conf. Intell. Robots and Systems, Hawaii, Oct. 29-Nov, 3, 2001.

[6] L. F. Hotraphinyo and C. N. Riviere, "Precision measurement for microsurgical instrument evaluation," Proc. 23rd Intl. Conf. IEEE Eng. Med. Biol. Soc., Istanbul, Oct. 2001, pp. 3454-3457.

[7] S. Charles, "Dexterity enhancement for surgery," in R. H. Taylor et al., eds., Computer integrated Surgery: Technology and Clinical Application. Cambridge: MIT Press, pp. 467-471, 1996. 